SCIPP $02 / 27$

November, 2002

hep-ph/0212010

\title{
Decoupling and the Radiatively-Corrected MSSM Higgs Sector
}

\author{
Howard E. Haber \\ Santa Cruz Institute for Particle Physics, \\ University of California, Santa Cruz, CA 95064
}

\begin{abstract}
In the decoupling limit of a non-minimal Higgs sector, the lightest CP-even Higgs boson $(h)$ is indistinguishable from the Standard Model (SM) Higgs boson. In the two-Higgs-doublet sector of the MSSM, the approach to the decoupling limit (for $\left.m_{A} \gg m_{Z}\right)$ persists, even in the presence of potentially large ( $\tan \beta$-enhanced) radiative corrections to the $h b \bar{b}$ coupling. Radiative corrections can also generate an accidental cancellation between tree-level and one-loop terms, resulting in a SM-like Higgs boson for moderate $m_{A}$ outside the decoupling regime.
\end{abstract}

Invited talk at the

6th International Symposium on Radiative Corrections:

Application of Quantum Field Theory to Phenomenology-RADCOR 2002

and 6th Zeuthen Workshop on Elementary Particle Theory:

Loops and Legs in Quantum Field Theory

September 8-13, 2002, Kloster Banz, Germany 


\title{
Decoupling and the radiatively-corrected MSSM Higgs sector *
}

\author{
Howard E. Haber ${ }^{a}$ \\ ${ }^{a}$ Santa Cruz Institute for Particle Physics, \\ University of California, Santa Cruz, CA 95064 USA
}

In the decoupling limit of a non-minimal Higgs sector, the lightest CP-even Higgs boson $(h)$ is indistinguishable from the Standard Model (SM) Higgs boson. In the two-Higgs-doublet sector of the MSSM, the approach to the decoupling limit (for $m_{A} \gg m_{Z}$ ) persists, even in the presence of potentially large (tan $\beta$-enhanced) radiative corrections to the $h b \bar{b}$ coupling. Radiative corrections can also generate an accidental cancellation between treelevel and one-loop terms, resulting in a SM-like Higgs boson for moderate $m_{A}$ outside the decoupling regime.

\section{Introduction}

Suppose a Higgs boson is discovered at the LHC, and its properties are observed to coincide (within experimental error) to those of the Standard Model (SM) Higgs boson. Moreover, suppose that evidence for supersymmetry is found, which suggests that in the minimal version of the model (MSSM), the observed Higgs boson is the lightest state of a two-Higgs-doublet model (2HDM). Finally, imagine that no evidence for the heavier Higgs states at the LHC is found. With precision measurements at a future high energy $e^{+} e^{-}$Linear Collider (LC), can one determine the mass scale of the heavier Higgs states?

At the LHC, more than one Higgs scalar of the MSSM will often be observed if $\tan \beta \gg 1$ (due to enhanced Higgs couplings to down-type fermions). But, there is also a substantial region of moderate $\tan \beta$ in which only the lightest CPeven Higgs scalar $(h)$ is observed [1]. If the properties of $h$ approximate those of the SM Higgs boson $\left(h_{\mathrm{SM}}\right)$, a program of precision Higgs measurements at the LC will play a critical role in elucidating the physics of Higgs bosons.

\section{Decoupling Limit of the 2HDM [ 2]}

Given a non-minimal Higgs sector, the decoupling limit corresponds to the parameter regime in which all but one CP-even neutral Higgs scalar

\footnotetext{
*This work was supported in part by the U.S. Department of Energy under the grant DE-FG03-92ER40689.
}

are significantly heavier than the $Z$. The properties of the lightest CP-even Higgs boson are nearly indistinguishable from those of the Standard Model (SM) Higgs boson. The decoupling limit is very general and exists in many multiHiggs models. The MSSM Higgs sector provides a well-motivated example for the decoupling limit and is the main focus of this work.

First, consider the general 2HDM. The most general scalar potential is given by:

$$
\begin{aligned}
& \mathcal{V}=m_{11}^{2} \Phi_{1}^{\dagger} \Phi_{1}+m_{22}^{2} \Phi_{2}^{\dagger} \Phi_{2}-\left[m_{12}^{2} \Phi_{1}^{\dagger} \Phi_{2}+\text { h.c. }\right] \\
& +\frac{1}{2} \lambda_{1}\left(\Phi_{1}^{\dagger} \Phi_{1}\right)^{2}+\frac{1}{2} \lambda_{2}\left(\Phi_{2}^{\dagger} \Phi_{2}\right)^{2}+\lambda_{3}\left(\Phi_{1}^{\dagger} \Phi_{1}\right)\left(\Phi_{2}^{\dagger} \Phi_{2}\right) \\
& +\lambda_{4}\left(\Phi_{1}^{\dagger} \Phi_{2}\right)\left(\Phi_{2}^{\dagger} \Phi_{1}\right)+\frac{1}{2}\left[\lambda_{5}\left(\Phi_{1}^{\dagger} \Phi_{2}\right)^{2}+\text { h.c. }\right] \\
& +\left\{\left[\lambda_{6}\left(\Phi_{1}^{\dagger} \Phi_{1}\right)+\lambda_{7}\left(\Phi_{2}^{\dagger} \Phi_{2}\right)\right] \Phi_{1}^{\dagger} \Phi_{2}+\text { h.c. }\right\} .
\end{aligned}
$$

For simplicity, we assume that there is no explicit (or spontaneous) CP violation. Since the ground state must preserve $\mathrm{U}(1)_{\mathrm{EM}}$, the scalar vacuum expectation values are $\left\langle\Phi_{i}^{0}\right\rangle \equiv v_{i} / \sqrt{2}$, with $\tan \beta \equiv v_{2} / v_{1}$ and $v^{2} \equiv v_{1}^{2}+v_{2}^{2}=(246 \mathrm{GeV})^{2}$.

Diagonalizing the CP-even Higgs squared-mass matrix yields two CP-even scalar eigenstates:

$$
\begin{aligned}
h & =-\left(\sqrt{2} \operatorname{Re} \Phi_{1}^{0}-v_{1}\right) s_{\alpha}+\left(\sqrt{2} \operatorname{Re} \Phi_{2}^{0}-v_{2}\right) c_{\alpha}, \\
H & =\left(\sqrt{2} \operatorname{Re} \Phi_{1}^{0}-v_{1}\right) c_{\alpha}+\left(\sqrt{2} \operatorname{Re} \Phi_{2}^{0}-v_{2}\right) s_{\alpha}, \quad(2)
\end{aligned}
$$

where $c_{\alpha} \equiv \cos \alpha, s_{\alpha} \equiv \sin \alpha$. The other Higgs scalars of the model include a CP-odd state, $A$, and a charged scalar pair, $H^{ \pm}$. The decoupling limit is defined as the limit of $m_{A} \gg m_{Z}$, assuming $\lambda_{i} \lesssim \mathcal{O}(1)$. One can show that this 
limit corresponds to taking $\beta-\alpha \rightarrow \pi / 2$; i.e., $\cos (\beta-\alpha) \rightarrow 0$. In the approach to the decoupling limit, one finds [2]:

$$
\begin{aligned}
& m_{A}^{2} \simeq v^{2}\left[\frac{\widehat{\lambda}}{c_{\beta-\alpha}}+\lambda_{A}-\frac{3}{2} \widehat{\lambda} c_{\beta-\alpha}\right], \\
& m_{h}^{2} \simeq v^{2}\left(\lambda-\widehat{\lambda} c_{\beta-\alpha}\right) \\
& m_{H}^{2} \simeq m_{A}^{2}+\left(\lambda-\lambda_{A}+\widehat{\lambda} c_{\beta-\alpha}\right) v^{2} \\
& m_{H^{ \pm}}^{2}=m_{A}^{2}+\frac{1}{2}\left(\lambda_{5}-\lambda_{4}\right) v^{2}
\end{aligned}
$$

where $c_{\beta-\alpha} \equiv \cos (\beta-\alpha)$ and

$$
\begin{gathered}
\lambda \equiv \lambda_{1} c_{\beta}^{4}+\lambda_{2} s_{\beta}^{4}+\frac{1}{2}\left(\lambda_{3}+\lambda_{4}+\lambda_{5}\right) s_{2 \beta}^{2} \\
+2 s_{2 \beta}\left(\lambda_{6} c_{\beta}^{2}+\lambda_{7} s_{\beta}^{2}\right), \\
\widehat{\lambda} \equiv \frac{1}{2} s_{2 \beta}\left[\lambda_{1} c_{\beta}^{2}-\lambda_{2} s_{\beta}^{2}-\left(\lambda_{3}+\lambda_{4}+\lambda_{5}\right) c_{2 \beta}\right] \\
-\lambda_{6} c_{\beta} c_{3 \beta}-\lambda_{7} s_{\beta} s_{3 \beta}, \\
\lambda_{A} \equiv c_{2 \beta}\left(\lambda_{1} c_{\beta}^{2}-\lambda_{2} s_{\beta}^{2}\right)+\left(\lambda_{3}+\lambda_{4}\right) s_{2 \beta}^{2} \\
-\lambda_{5} c_{2 \beta}^{2}+2 \lambda_{6} c_{\beta} s_{3 \beta}-2 \lambda_{7} s_{\beta} c_{3 \beta} .
\end{gathered}
$$

In particular, eqs. (3)-(5) yield

$$
\cos (\beta-\alpha) \simeq \frac{\widehat{\lambda} v^{2}}{m_{A}^{2}-\lambda_{A} v^{2}} \simeq \frac{\widehat{\lambda} v^{2}}{m_{H}^{2}-m_{h}^{2}} .
$$

It follow that: (i) $m_{h} \sim \mathcal{O}\left(m_{Z}\right)$, (ii) $m_{H} \simeq m_{A} \simeq$ $m_{H^{ \pm}}$, up to corrections of $\mathcal{O}\left(m_{Z}^{2} / m_{A}\right)$, and (iii) $\cos (\beta-\alpha) \sim \mathcal{O}\left(m_{Z}^{2} / m_{A}^{2}\right)$.

The couplings of the Higgs bosons to vector bosons, fermions and scalars typically depend on $\alpha$ and $\beta$. By examining the tree-level couplings of the lightest CP-even scalar $h$, one notes that in the limit of $c_{\beta-\alpha}=0$, the couplings of $h$ reduce to the corresponding SM Higgs couplings. That is, in the approach to the decoupling limit, the properties of $h$ are nearly indistinguishable from those of the SM Higgs boson, whereas all the other Higgs states are significantly heavier. Thus, the effective low energy theory below the mass scale of $\mathcal{O}\left(m_{A}\right)$ is the Standard Model with one Higgs doublet.

The Higgs sector of the minimal supersymmetric extension of the Standard Model (MSSM) is a two-Higgs doublet model, with interactions constrained by supersymmetry [ [3]. It particular,

$$
\begin{array}{ll}
\lambda v^{2}=m_{Z}^{2} c_{2 \beta}^{2}, & \lambda_{4} v^{2}=-2 m_{W}^{2}, \\
\widehat{\lambda} v^{2}=m_{Z}^{2} s_{2 \beta} c_{2 \beta}, & \lambda_{A} v^{2}=m_{Z}^{2} c_{4 \beta},
\end{array}
$$

and $\lambda_{5}=\lambda_{6}=\lambda_{7}=0$. Moreover, the following tree-level result can be derived:

$$
\cos ^{2}(\beta-\alpha)=\frac{m_{h}^{2}\left(m_{Z}^{2}-m_{h}^{2}\right)}{m_{A}^{2}\left(m_{H}^{2}-m_{h}^{2}\right)} .
$$

As expected, $c_{\beta-\alpha} \rightarrow 0$ in the decoupling limit where $m_{h} \sim \mathcal{O}\left(m_{Z}\right)$ and $m_{A} \gg m_{Z}$. Moreover, when $m_{A} \gg m_{Z}$, eqs. (3)-(6) yield

$$
\begin{aligned}
& m_{h}^{2} \simeq m_{Z}^{2} c_{2 \beta}^{2}, \quad m_{H}^{2} \simeq m_{A}^{2}+m_{Z}^{2} s_{2 \beta}^{2}, \\
& m_{H^{ \pm}}^{2}=m_{A}^{2}+m_{W}^{2}, \quad c_{\beta-\alpha} \simeq \frac{m_{Z}^{4} \sin ^{2} 4 \beta}{4 m_{A}^{4}} .
\end{aligned}
$$

\section{A SM-like Higgs boson without decou- pling [2]}

It is possible for the theory to exhibit a SM-like Higgs boson without decoupling. For example, assume that $\tan \beta \geq 1$. Two cases arise in the $2 \mathrm{HDM}$ where $\left|c_{\beta-\alpha}\right| \ll 1$ : (i) $m_{A}^{2} \gg \lambda_{i} v^{2} \tan \beta$, with $\lambda_{i} \lesssim \mathcal{O}(1)$, and (ii) $|\widehat{\lambda}| \ll 1$ with $m_{A}$ arbitrary. (If $\tan \beta \leq 1$, replace $\tan \beta$ with $\cot \beta$ above.) In the MSSM, the $h b \bar{b}$ coupling normalized to its SM value is given by:

$$
-\frac{\sin \alpha}{\cos \beta}=\sin (\beta-\alpha)-\tan \beta \cos (\beta-\alpha) .
$$

In case (i), we have $\left|\tan \beta c_{\beta-\alpha}\right| \ll 1$ even if $\tan \beta \gg 1$ [see eq. (10)], which implies that the $h b \bar{b}$ coupling is SM-like (corresponding to the decoupling limit). Case (ii) does not correspond to decoupling if $m_{A}^{2} \lesssim \mathcal{O}\left(v^{2}\right)$. If $\tan \beta \gg 1$, it is possible to have $\left|\tan \beta c_{\beta-\alpha}\right| \sim \mathcal{O}(1)$ even if $\left|c_{\beta-\alpha}\right| \ll 1$, in which case the $h b \bar{b}$ coupling deviates from its $\mathrm{SM}$ value. Nevertheless, for the Higgs couplings to $t \bar{t}$, vector bosons and scalars, $h$ is SM-like.

In the MSSM at tree-level, $\left|c_{\beta-\alpha}\right| \ll 1$ is possible only in the decoupling regime [see eq. (12)], corresponding to case (i) above. However, oneloop effects mediated by supersymmetric particles can generate significant modifications to the tree-level MSSM Higgs sector. For example, the tree-level upper bound, $m_{h} \leq m_{Z}$, can be significantly raised [ 4]. Allowing for maximal mixing in the top squark sector and supersymmetric mass parameters of order $M_{S} \sim 1 \mathrm{TeV}$, one finds a radiatively-corrected Higgs mass bound of 
$m_{h} \lesssim 135 \mathrm{GeV}$ [ 1]. We shall demonstrate in section 4 that for moderate values of $m_{A}$, radiative corrections to $\widehat{\lambda}$ can result in $|\widehat{\lambda}| \ll 1$ in certain regions of the MSSM parameter space. This would correspond to case (ii) above, and the $h$ of the MSSM could indeed exhibit SM-like properties outside the domain of the decoupling limit.

\section{Radiatively-corrected MSSM Higgs couplings [ 9]}

In order to study the decoupling properties of the MSSM Higgs sector, it is crucial to examine the Higgs couplings, including the most significant loop-corrections. The leading contributions to the radiatively-corrected Higgs couplings arise in two ways. First, the radiative corrections to the CP-even Higgs squared-mass matrix results in a shift of the CP-even Higgs mixing angle $\alpha$ from its tree-level value. That is, the dominant Higgs propagator corrections can to a good approximation be absorbed into an effective ("radiativelycorrected") mixing angle $\alpha$ [5]. In this approximation, we can write:

$\mathcal{M}^{2} \equiv\left(\begin{array}{ll}\mathcal{M}_{11}^{2} & \mathcal{M}_{12}^{2} \\ \mathcal{M}_{12}^{2} & \mathcal{M}_{22}^{2}\end{array}\right)=\mathcal{M}_{0}^{2}+\delta \mathcal{M}^{2}$,

where the tree-level contribution is denoted by $\mathcal{M}_{0}^{2}$ and $\delta \mathcal{M}^{2}$ is the contribution from the radiative corrections. Then, $c_{\beta-\alpha}$ is given by

$c_{\beta-\alpha}=\frac{\left(\mathcal{M}_{11}^{2}-\mathcal{M}_{22}^{2}\right) \sin 2 \beta-2 \mathcal{M}_{12}^{2} \cos 2 \beta}{2\left(m_{H}^{2}-m_{h}^{2}\right) \sin (\beta-\alpha)}$.

Inserting the tree-level values for $\mathcal{M}_{0}^{2}$, one can rewrite eq. (16) as

$$
\begin{aligned}
& c_{\beta-\alpha}=\frac{m_{Z}^{2} \sin 4 \beta}{2\left(m_{H}^{2}-m_{h}^{2}\right) \sin (\beta-\alpha)} \\
& +\frac{\left(\delta \mathcal{M}_{11}^{2}-\delta \mathcal{M}_{22}^{2}\right) \sin 2 \beta-2 \delta \mathcal{M}_{12}^{2} \cos 2 \beta}{2\left(m_{H}^{2}-m_{h}^{2}\right) \sin (\beta-\alpha)} .
\end{aligned}
$$

Using tree-level Higgs couplings with $\alpha$ replaced by its effective one-loop value provides a useful first approximation to the radiatively-corrected Higgs couplings.

Second, contributions from the one-loop vertex corrections to tree-level Higgs-fermion couplings can modify these couplings in a significant way, especially in the limit of large $\tan \beta$.
When radiative corrections are included, all possible dimension-four Higgs-fermion couplings are generated. In particular, the effects of higher dimension operators can be ignored if $M_{S} \gg m_{Z}$, which we henceforth assume. These results can be summarized by an effective Lagrangian that describes the coupling of the neutral Higgs bosons to the third generation quarks:

$$
\begin{aligned}
-\mathcal{L}_{\text {eff }} & =\left(h_{b}+\delta h_{b}\right) \bar{b}_{R} b_{L} \Phi_{1}^{0 *}+\left(h_{t}+\delta h_{t}\right) \bar{t}_{R} t_{L} \Phi_{2}^{0} \\
& +\Delta h_{t} \bar{t}_{R} t_{L} \Phi_{1}^{0}+\Delta h_{b} \bar{b}_{R} b_{L} \Phi_{2}^{0 *}+\text { h.c. }, \quad(18)
\end{aligned}
$$

resulting in a modification of the tree-level relation between $h_{t}\left[h_{b}\right]$ and $m_{t}\left[m_{b}\right]$ as follows [ 6, 8, 8):

$$
\begin{gathered}
m_{b}=\frac{h_{b} v}{\sqrt{2}} \cos \beta\left(1+\frac{\delta h_{b}}{h_{b}}+\frac{\Delta h_{b} \tan \beta}{h_{b}}\right) \\
\equiv \frac{h_{b} v}{\sqrt{2}} \cos \beta\left(1+\Delta_{b}\right), \\
m_{t}=\frac{h_{t} v}{\sqrt{2}} \sin \beta\left(1+\frac{\delta h_{t}}{h_{t}}+\frac{\Delta h_{t} \cot \beta}{h_{t}}\right) \\
\equiv \frac{h_{t} v}{\sqrt{2}} \sin \beta\left(1+\Delta_{t}\right) .
\end{gathered}
$$

The dominant contributions to $\Delta_{b}$ are $\tan \beta$ enhanced, with $\Delta_{b} \simeq\left(\Delta h_{b} / h_{b}\right) \tan \beta$; whereas for $\tan \beta \gg 1, \delta h_{b} / h_{b}$ provides a small correction to $\Delta_{b}$. [In the same limit, $\Delta_{t} \simeq \delta h_{t} / h_{t}$, with the additional contribution of $\left(\Delta h_{t} / h_{t}\right) \cot \beta$ providing a small correction.]

From eq. (18) we can obtain the couplings of the physical neutral Higgs bosons to third generation quarks. The resulting couplings of $h$ to $b \bar{b}$ and $t \bar{t}$ pairs are given by:

$$
\begin{aligned}
& g_{h t \bar{t}}= \frac{m_{t}}{v} \frac{\cos \alpha}{\sin \beta}\left[1-\frac{1}{1+\Delta_{t}} \frac{\Delta h_{t}}{h_{t}}(\cot \beta+\tan \alpha)\right] \\
& g_{h b \bar{b}}=-\frac{m_{b}}{v} \frac{\sin \alpha}{\cos \beta}\left[1+\frac{1}{1+\Delta_{b}}\right. \\
&\left.\quad \times\left(\frac{\delta h_{b}}{h_{b}}-\Delta_{b}\right)(1+\cot \alpha \cot \beta)\right] .(21)
\end{aligned}
$$

We now turn to the decoupling limit. First consider the implications for the radiativelycorrected value of $c_{\beta-\alpha}$. Since $\delta \mathcal{M}_{i j}^{2} \sim \mathcal{O}\left(m_{Z}^{2}\right)$, and $m_{H}^{2}-m_{h}^{2}=m_{A}^{2}+\mathcal{O}\left(m_{Z}^{2}\right)$, one finds [ [9]

$\cos (\beta-\alpha)=c\left[\frac{m_{Z}^{2} \sin 4 \beta}{2 m_{A}^{2}}+\mathcal{O}\left(\frac{m_{Z}^{4}}{m_{A}^{4}}\right)\right]$, 
in the limit of $m_{A} \gg m_{Z}$, where

$c \equiv 1+\frac{\delta \mathcal{M}_{11}^{2}-\delta \mathcal{M}_{22}^{2}}{2 m_{Z}^{2} \cos 2 \beta}-\frac{\delta \mathcal{M}_{12}^{2}}{m_{Z}^{2} \sin 2 \beta}$.

Equivalently, the radiative corrections have modified the tree-level definition of $\widehat{\lambda}$ :

$\widehat{\lambda} v^{2}=c m_{Z}^{2} \sin 2 \beta \cos 2 \beta$.

Eq. (22) exhibits the expected decoupling behavior for $m_{A} \gg m_{Z}$. However, eqs. (17) and (22) exhibit another way in which $\cos (\beta-\alpha)=0$ can be achieved - simply choose the MSSM parameters (which govern the Higgs mass radiative corrections) such that the right hand side of eq. (17) vanishes. That is,

$$
\sin 2 \beta=\frac{2 \delta \mathcal{M}_{12}^{2}-\tan 2 \beta\left(\delta \mathcal{M}_{11}^{2}-\delta \mathcal{M}_{22}^{2}\right)}{2 m_{Z}^{2}} .
$$

This condition is equivalent to $c=0$, which implies $\widehat{\lambda}=0$ [eq. (24)] and thus corresponds to case (ii) in section 3. That is, although $\widehat{\lambda} \neq 0$ at treelevel, the one-loop radiative corrections to $\widehat{\lambda}$ can approximately cancel its tree-level value, resulting in $|\widehat{\lambda}| \ll 1$. (Note that the one-loop corrections arise from the exchange of supersymmetric particles, whose contributions can be enhanced for certain MSSM parameter choices. One can show that the two-loop corrections are subdominant, so that the approximation scheme is under control.) In particular, eq. (25) is independent of the value of $m_{A}$. Typically, eq. (25) yields a solution at large $\tan \beta$. That is, by approximating $\tan 2 \beta \simeq-\sin 2 \beta \simeq-2 / \tan \beta$, one can determine the value of $\beta$ at which $\widehat{\lambda} \simeq 0[9]$ :

$\tan \beta \simeq \frac{2 m_{Z}^{2}-\delta \mathcal{M}_{11}^{2}+\delta \mathcal{M}_{22}^{2}}{\delta \mathcal{M}_{12}^{2}}$

Hence, there exists a value of $\tan \beta$ (which depends on the choice of MSSM parameters) where $\cos (\beta-\alpha) \simeq 0$ independently of the value of $m_{A}$. If $m_{A}$ is not much larger than $m_{Z}$, then $h$ is a SMlike Higgs boson outside the decoupling regime.

Finally, note that for $m_{A} \gg m_{Z}$,

$\cot \alpha=-\tan \beta\left[1+\frac{2 m_{Z}^{2}}{m_{A}^{2}} c_{2 \beta}\right]+\mathcal{O}\left(\frac{m_{Z}^{4}}{m_{A}^{4}}\right)$
Applying this result to eq. (21), it follows that in the decoupling limit, $g_{h q \bar{q}}=g_{h_{\mathrm{SM}} q \bar{q}}=m_{q} / v$. Away from the decoupling limit, the Higgs couplings to down-type fermions can deviate significantly from their tree-level values due to enhanced radiative corrections at large $\tan \beta$ [where $\left.\Delta_{b} \simeq \mathcal{O}(1)\right]$. In particular, because $\Delta_{b} \propto \tan \beta$, the leading one-loop radiative correction to $g_{h b \bar{b}}$ is of $\mathcal{O}\left(m_{Z}^{2} \tan \beta / m_{A}^{2}\right)$, which decouples only when $m_{A}^{2} \gg m_{Z}^{2} \tan \beta$ (this behavior was called delayed decoupling in [10]).

\section{Implications for precision Higgs mea- surements at the $\mathrm{LC}$}

As noted in section 1, a program of precision Higgs measurements at the LC may be critical in determining whether the properties of the lightest CP-even Higgs boson differ from those of $h_{\mathrm{SM}}$. In particular, recent simulations of Higgs branching ratio measurements [11] suggest that the Higgs couplings to vector bosons and the third generation fermions can be determined with an accuracy in the range of $1-3 \%$ at the LC. In the exact decoupling limit (of infinitely large $m_{A}$ ), $h=h_{\mathrm{SM}}$. However, for finite values of $m_{A}$, the fractional deviations of the couplings of $h$ relative to those of $h_{\mathrm{SM}}$ scale as $m_{Z}^{2} / m_{A}^{2}$. Thus, if precision measurements reveal a significant deviation from SM expectations, one could in principle derive a constraint (e.g., upper and lower bounds) on the heavy Higgs masses of the model.

In the MSSM, this constraint is sensitive to the supersymmetric parameters that control the radiative corrections to the Higgs couplings. This is illustrated in fig. 11, where the constraints on $m_{A}$ are derived for two different sets of MSSM parameter choices [ 9]. Here, a simulation of a global fit of measured $h b b, h \tau \tau$ and $h g g$ couplings is made (based on the anticipated experimental accuracies given in [11]) and $\chi^{2}$ contours are plotted indicating the constraints in the $m_{A}-\tan \beta$ plane, assuming that a deviation from SM Higgs boson couplings is seen. In the maximal mixing scenario shown in fig. 1 (a), the constraints on $m_{A}$ are significant and rather insensitive to the value of $\tan \beta$. However in some cases, as shown in fig. 11(b), a region of $\tan \beta$ may yield almost 

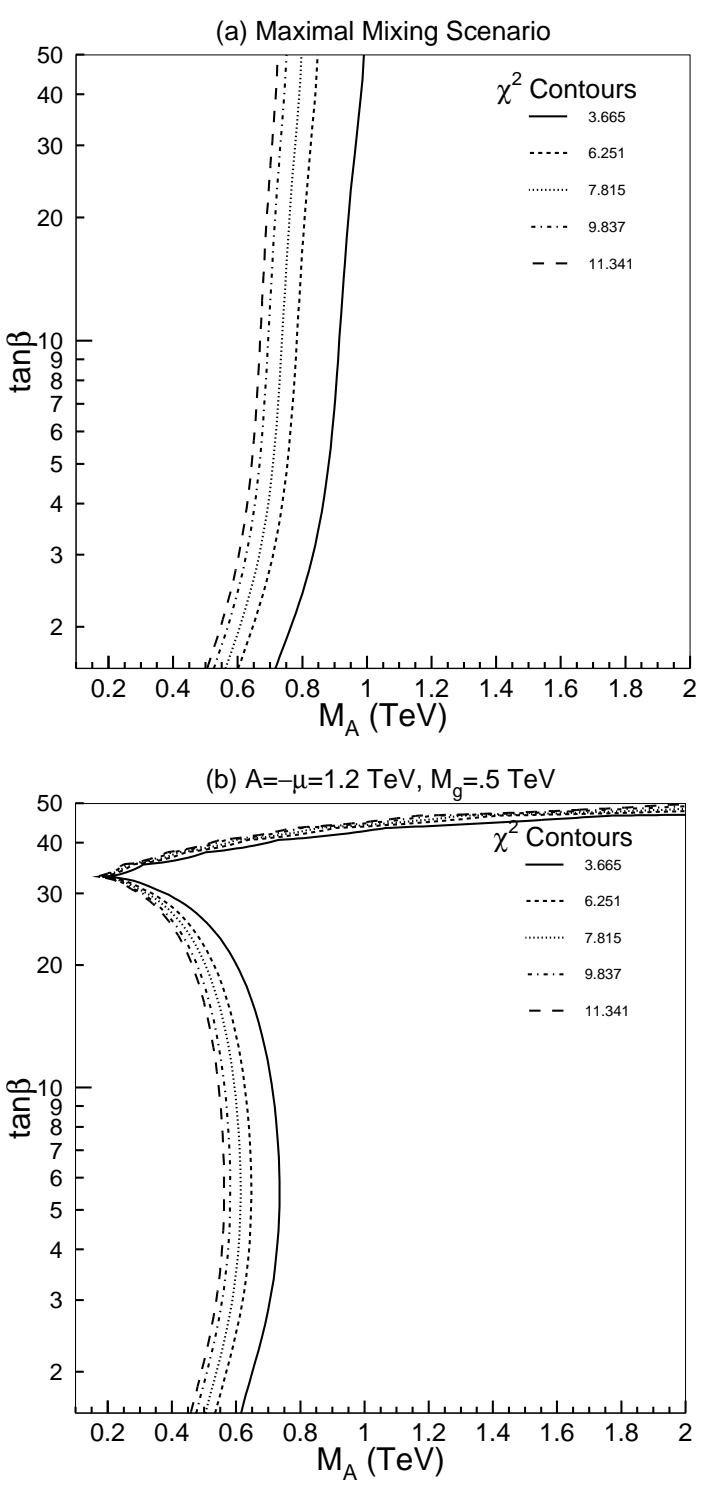

Figure 1. Contours of $\chi^{2}$ for Higgs boson decay observables for (a) the maximal mixing scenario; and (b) a choice of MSSM parameters for which the loop-corrected $h b \bar{b}$ coupling is suppressed at large $\tan \beta$ and low $m_{A}$ (relative to the corresponding tree-level coupling). The contours correspond to $68,90,95,98$ and $99 \%$ confidence levels (right to left) for the observables $g_{h b b}^{2}, g_{h \tau \tau}^{2}$, and $g_{h g g}^{2}$. See [9] for additional details. no constraint on $m_{A}$. This corresponds to the value of $\tan \beta$ given by eq. (26), and is a result of $\widehat{\lambda} \simeq 0$ generated by radiative corrections $[c \simeq 0$ in eq. (24)]. Thus, one cannot extract a fully model-independent upper bound on the value of $m_{A}$ beyond the kinematical limit that would be obtained if direct $A$ production were not observed at the LC.

\section{REFERENCES}

1. For a review and references, see M. Carena and H.E. Haber, FERMILAB-Pub-02/114-T and SCIPP 02/07 hep-ph/0208209].

2. J.F. Gunion and H.E. Haber, UCD-2002-10 and SCIPP-02/10 hep-ph/0207010.

3. K. Inoue, A. Kakuto, H. Komatsu, and S. Takeshita, Prog. Theor. Phys. 67 (1982) 1889; R. Flores and M. Sher, Annals Phys. 148 (1983) 95; J.F. Gunion and H.E. Haber, Nucl. Phys. B272 (1986) 1 [E: B402 (1993) $567]$.

4. H.E. Haber and R. Hempfling, Phys. Rev. Lett. 66 (1991) 1815; Y. Okada, M. Yamaguchi and T. Yanagida, Prog. Theor. Phys. 85 (1991) 1; J.R. Ellis, G. Ridolfi and F. Zwirner, Phys. Lett. B257 (1991) 83.

5. S. Heinemeyer, W. Hollik and G. Weiglein, Eur. Phys. J. C16 (2000) 139.

6. R. Hempfling, Phys. Rev. D49 (1994) 6168; L.J. Hall, R. Rattazzi and U. Sarid, Phys. Rev. D50 (1994) 7048; M. Carena, M. Olechowski, S. Pokorski and C.E.M. Wagner, Nucl. Phys. B426 (1994) 269.

7. A. Bartl, et al., Phys. Lett. B378 (1996) 167; R.A. Jiménez and J. Solà, Phys. Lett. B389 (1996) 53; J.A. Coarasa, R.A. Jiménez and J. Solà, Phys. Lett. B389 (1996) 312.

8. D.M. Pierce, J.A. Bagger, K. Matchev, and R. Zhang, Nucl. Phys. B491 (1997) 3.

9. M. Carena, H.E. Haber, H.E. Logan and S. Mrenna, Phys. Rev. D65 (2002) 055005 [E: D65 (2002) 099902].

10. H.E. Haber, M.J. Herrero, H.E. Logan, S. Peñaranda, S. Rigolin and D. Temes, Phys. Rev. D63 (2001) 055004.

11. M. Battaglia and K. Desch, hep-ph/0101165. 